\title{
A CCD area detector for X-ray diffraction under high pressure for rotating anode source ${ }^{\dagger}$
}

\author{
AMAR SINHA, ALKA B GARG*, V VIJAYAKUMAR, B K GODWAL and S K SIKKA \\ High Pressure Physics Division, Bhabha Atomic Research Centre, Mumbai 400 085, India
}

\begin{abstract}
Details of a two-dimensional X-ray area detector developed using a charge coupled device, a image intensifier and a fibre optic taper are given. The detector system is especially optimized for angle dispersive $\mathrm{X}$-ray diffraction set up using rotating anode generator as X-ray source. The performance of this detector was tested by successfully carrying out powder $\mathrm{X}$-ray diffraction measurements on various materials such as intermetallics $A u n_{2}, A u G a$, high $Z$ material $P d$ and low $Z$ scatterer adamantane $\left(\mathrm{C}_{10} \mathrm{H}_{16}\right)$ at ambient conditions. Its utility for quick detection of phase transitions at high pressures with diamond anvil cell is demonstrated by reproducing the known pressure induced structural transitions in RbI, KI and a new structural phase transition in $\mathrm{AuGa}_{2}$ above $10 \mathrm{GPa}$. Various softwares have also been developed to analyze data from this detector.
\end{abstract}

Keywords. CCD X-ray detector; X-ray diffraction; high pressure studies.

\section{Introduction}

The high sensitivity, wide dynamic range and high spatial resolution of image plate (IP) area detector are currently being exploited for investigation of materials under pressure to study phase transitions and equation of state. However, because of the small sample size in the high pressure diamond anvil cell (DAC), the recording time of the diffraction patterns with IP, is still a few hours with rotating anode X-ray source. The reduction in acquisition time is thus an important task. For this, various laboratories in the world are developing the charge coupled device (CCD) (Deacan et al 1990; Gago et al 1993; Hamaya et al 1996) based area detectors. These detectors show all the promising features of imaging plates combined with quick data collection ability. The availability of intense X-rays from synchrotron sources has opened up possibilities of real time investigations of materials in DAC using X-ray diffraction with $\mathrm{CCD}$ as area detector. However, the high cost of these detectors have limited their utilization (Fuchs et al 1990).

We have designed a low cost prototype CCD based area detector suited for rotating anode generator for fast data collection at normal and high pressure conditions for angle dispersive powder diffraction (ADXRD) studies and to provide definite directions in which the more accurate experiments employing IP can be carried out.

The performance of this detector system is established by carrying out angle dispersive X-ray diffraction measurements

\footnotetext{
*Author for correspondence

${ }^{\dagger}$ Paper presented at the 5th IUMRS ICA98, October 1998, Bangalore
}

on various substances like high atomic number element Pd, intermetallics like $\mathrm{AuIn}_{2}, \mathrm{AuGa}_{2}$ and low scatterer like molecular solid adamantane $\mathrm{C}_{10} \mathrm{H}_{16}$ at ambient conditions. Its utility for high pressure $\mathrm{X}$-ray diffraction studies has been tested by reproducing the known pressure induced structural phase transitions in RbI and KI (Vaidya and Kennedy 1971). We have also used it for quick check of an unknown structural phase transition in the intermetallic compound $\mathrm{AuGa}_{2}$.

\section{Description of detector}

The schematic diagram of the detector is shown in figure 1 . The main parameters guiding the selection of components for designing such a CCD based detector system have been optical collection efficiency, optical demagnification, resolution, detection efficiency of the scintillating screen and its thickness. The detector uses a X-ray sensitive scintillator pasted on a taper fibre optic having input area of $70 \mathrm{~mm}$ coupled to a two-stage image intensifier tube. The output of image intensifier tube is transferred through a lens to a CCD imaging camera. The overall gain of the image intensifiers system which consists of two lens coupled image intensifiers can be manually adjusted depending on X-ray intensity. The two second generation image intensifier tubes have input/output diameter of $18 \mathrm{~mm}$ with $30 \mathrm{lp} / \mathrm{mm}$ spatial resolution (Sinha et al 1996). The CCD employed by us is $2 / 3$ inch in diameter having $756(\mathrm{H}) \times 581(\mathrm{~V})$ pixels with pixel size of $11.0 \mu \mathrm{m}(\mathrm{H}) \times 11.0 \mu \mathrm{m}(\mathrm{V})$. The video signals obtained from CCD are digitized using a 8 bit frame grabber with a 16 bit processor. We have introduced provision for online thresholding and online dark current subtraction. Shading 
correction to correct for the systematic nonuniformity of detector response was also incorporated. The image acquisition system can display real time images on a separate video monitor $(40 \mathrm{~ms})$ or display background subtracted images or integrated images using 16 bit onboard processor. We have also developed two custom designed algorithms to analyze the X-ray diffraction patterns. The first one iteratively determines the pixel position of the centre of the diffraction rings. The second algorithm carries out radial integration along the Debye-Sherrer rings.

\section{Experimental}

In order to study the performance of CCD area detector we have used it in place of IP detector in our ADXRD system (Godwal et al 1997). We used rotating anode Xray generator operating at $50 \mathrm{KV}$ and $40 \mathrm{~mA}$ to generate the X-rays. Zirconium filtered X-rays were collimated to $300 \mu \mathrm{m}$ size at the samples. The overall resolution (pixel size) of the detector was $170 \mu \mathrm{m}$. In order to turn the radially integrated digitized image into a standard onedimensional (1-D) powder pattern expressed in terms of $2 \theta$, the sample to detector distance was accurately determined $(\sim 10 \mathrm{~cm})$ by collecting the diffraction pattern of $\mathrm{Pd}$ at ambient conditions and from the knowledge of its lattice parameter (Pearson 1967).

\section{Results and discussion}

The typical patterns are shown for $\mathrm{Pd}$ and $\mathrm{AuGa}_{2}$ in figures $2 \mathrm{a}-\mathrm{b}$. The strong powder diffraction rings from good scatterers like $\mathrm{Pd}$ and the intermetallics such as $\mathrm{AuIn}_{2}$, and $\mathrm{AuGa}_{2}$ were in fact observed at real time rate of $40 \mathrm{~ms}$. The texture is seen in diffraction rings of $\mathrm{Pd}$. Radially integrated diffraction patterns after background subtraction were transformed to one-dimensional pattern using the procedure described in $\S 3$. In figures $3 a-b$, we display these diffraction patterns for $\mathrm{AuGa}_{2}$ and KI. The data was fitted to yield the ambient values of the lattice

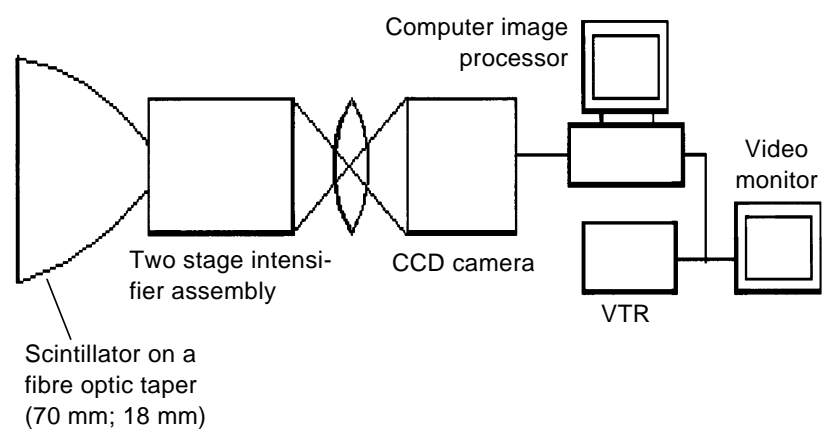

Figure 1. Schematic diagram of CCD based area detector for angle dispersive $\mathrm{X}$-ray diffraction (ADXRD) experiments. parameters of $\mathrm{AuGa}_{2}$ and $\mathrm{KI}$, respectively. An angular range of $2 \theta$ equal to $40^{\circ}$ has been covered and if required, a step scan can be used to increase this to a higher desired value. A Rietveld refinement of all powder data with pseudo Voigt line profile yielded good agreement between the observed and simulated patterns (Rietveld 1967, 1969). In figure 4 we illustrate it for $\mathrm{AuGa}_{2}$. The refined value of the lattice parameter for $\mathrm{AuGa}_{2}$ was found to be

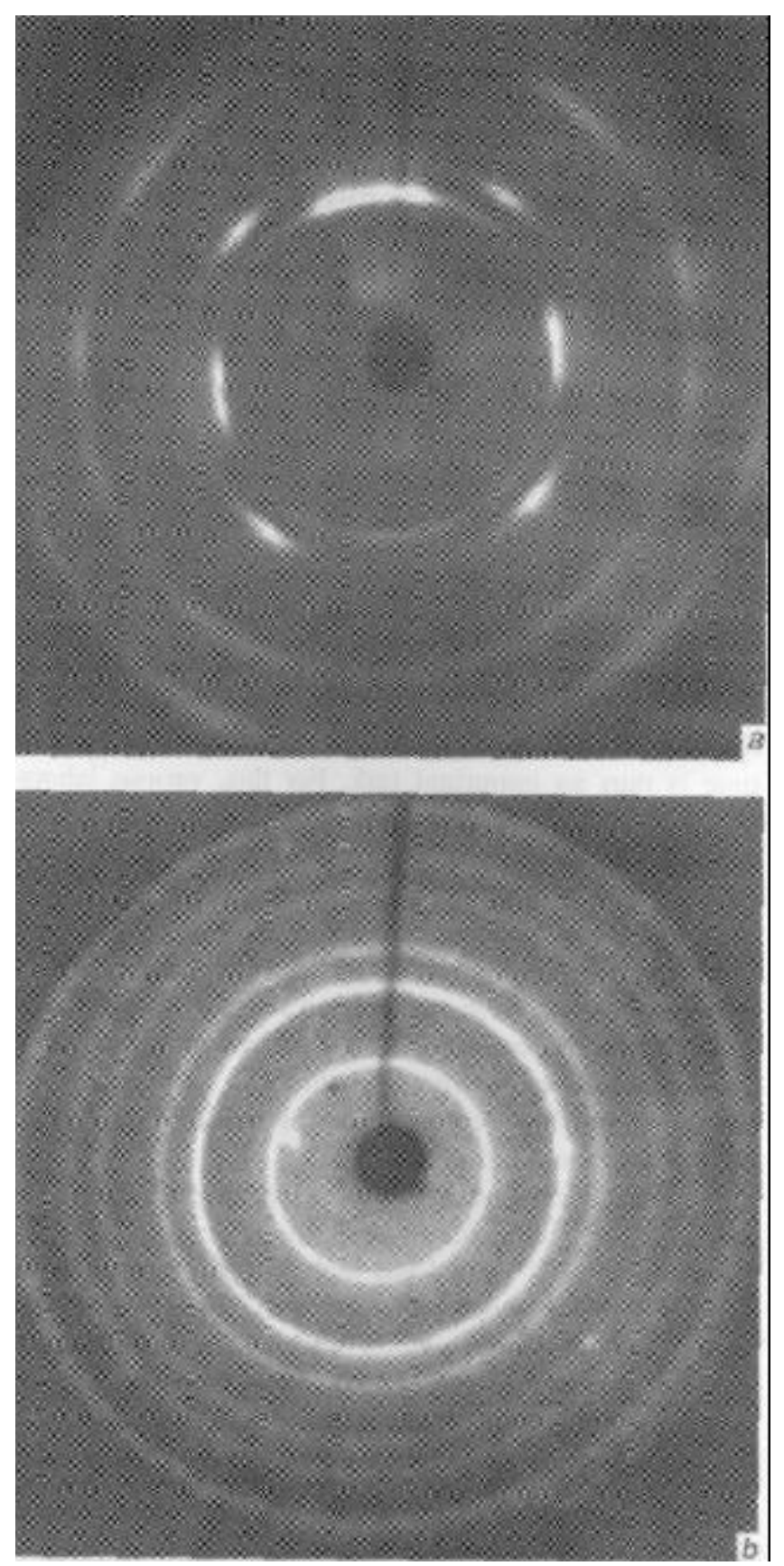

Figure 2. Online Debye-Sherrer rings for (a) palladium and (b) $\mathrm{AuGa}_{2}$. 
6.050 А which can be compared with the published value (Pearson 1967) of 6.07.

For high pressure measurements, RbI, KI and $\mathrm{AuGa}_{2}$ samples were loaded in a modified Mao Bell diamond anvil cell (DAC) along with a ruby chip (Chidambaram
1992). Pressure was measured by ruby fluorescence technique (Mao et al 1996). The diffracted X-rays at various pressures were recorded on the $\mathrm{CCD}$ detector placed behind the DAC. In view of the limited opening of the $\mathrm{DAC}$, the angular range $\left(40^{\circ}\right)$ of the detector does not
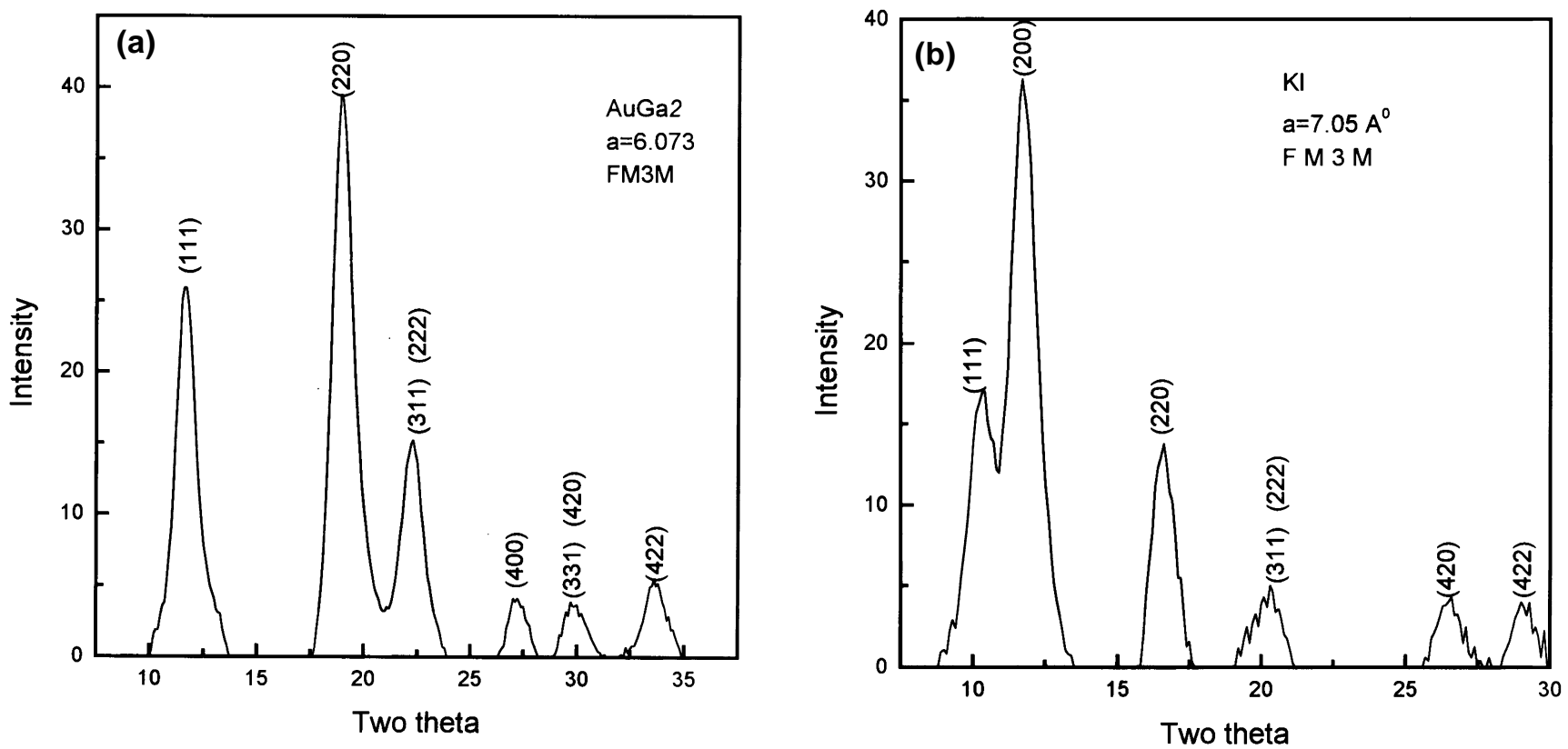

Figure 3. Radially integrated and background subtracted 1-D patterns for (a) $\mathrm{AuGa}_{2}$ and (b) KI. The space groups and refined values of lattice parameters are also shown.

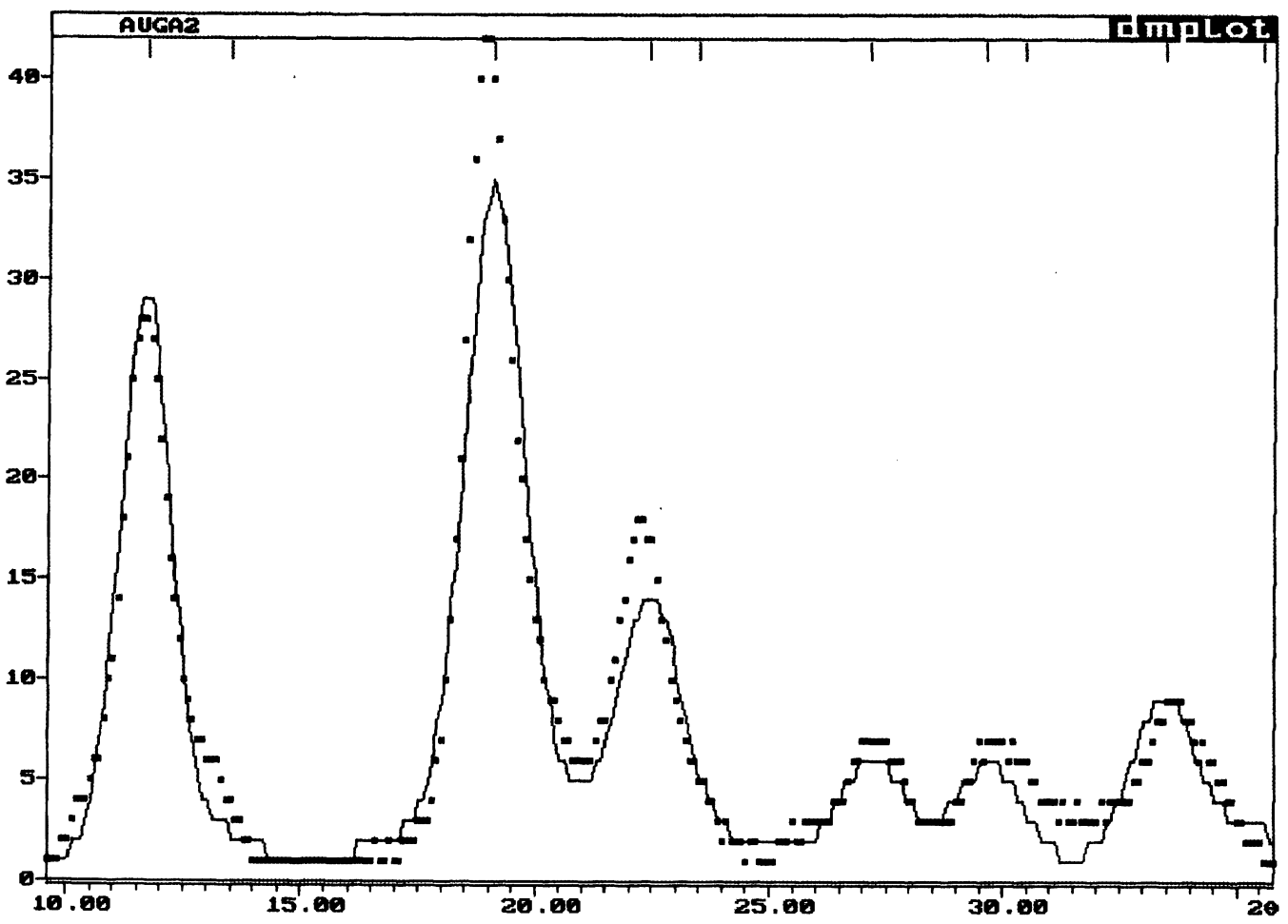

Figure 4. Rietveld refined pattern for $\mathrm{AuGa}_{2}$. 


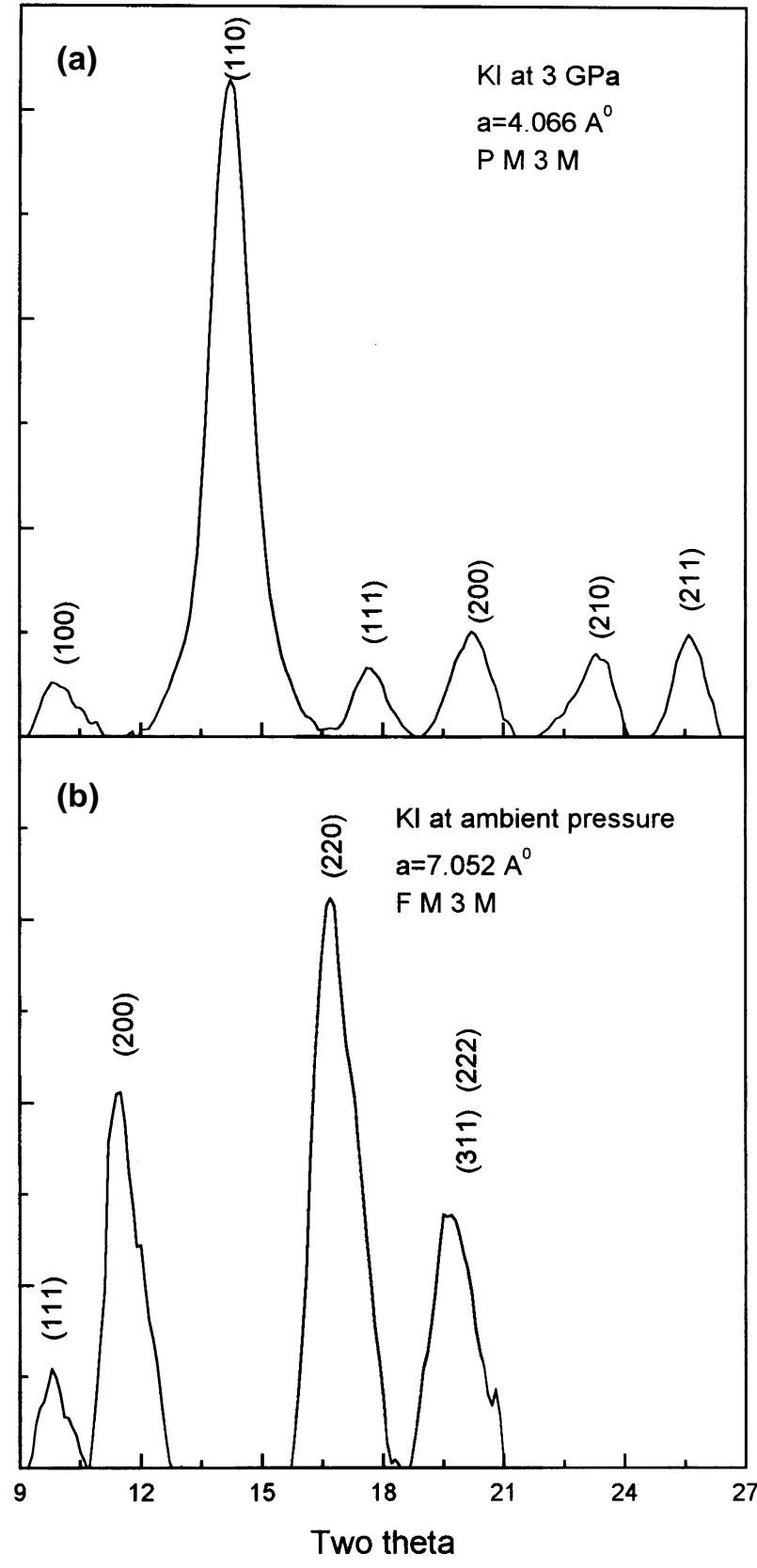

Figure 5. (a) 1-D high pressure (3 GPa) X-ray diffraction pattern for KI. The indexing of various lines as seen in the figure is consistent with $\mathrm{CsCl}$ structure. (b) X-ray diffraction pattern for $\mathrm{KI}$ at ambient pressure in $\mathrm{NaCl}$ phase.

limit its utility for high pressure measurements. In figure 5 we show the one-dimensional diffraction patterns of $\mathrm{KI}$ when the pressure was increased to about $3.0 \mathrm{GPa}$. It is evident from the figure that $\mathrm{KI}$ has transformed to the $\mathrm{CsCl}$ structure from its ambient $\mathrm{NaCl}$ structure (Vaidya and Kennedy 1971). The measured value of the lattice parameter of $4.066 \AA$ at $3 \mathrm{GPa}$ pressure is consistent with the known experimental data (Vaidya and Kennedy 1971). On release of pressure $\mathrm{KI}$ returned to its ambient $\mathrm{NaCl}$ structure.
We have also used this system to look for quick signatures of the structural phase transition in the intermetallic compound $\mathrm{AuGa}_{2}$ to the pressures of about $10 \mathrm{GPa}$ (Alka et al 1998). The present high pressure X-ray diffraction measurements carried out using the CCD based X-ray imaging reveal the splitting of [200] line of the $\mathrm{CaF}_{2}$ phase above $10 \mathrm{GPa}$ similar to that observed by us in $\mathrm{AuIn}_{2}$ (Godwal et al 1997). The subsequent detailed measurements carried out using IP has indeed confirmed this.

\section{Conclusions}

The performance of a CCD based area detector system developed by us is demonstrated at ambient and high pressure conditions using low current rotating anode $\mathrm{X}$ ray source. The feasibility of reducing the data acquisition times of few hours (for e.g. while using IP area detector) to few tens of seconds is clearly established by collecting diffraction data, which can be Rietveld, refined. Considering the variety of samples and experiments for which this detector has been tested, it can be inferred that such detectors can play a vital role in rapid scan of various phase transitions in materials and provide the definite directions in which the more accurate experiments employing IP can be carried out. This detector may also be suited for certain new class of experiments such as time resolved phase transitions which are not feasible with the present form of an IP area detector. If the current CCD is replaced by a cooled one then higher dynamic range and precision can be obtained.

\section{References}

Alka B Garg, Vijayakumar V, Rao R S, Sinha A, Godwal B K and Sikka S K 1998 Phys. Rev. Lett. (under preparation)

Chidambaram R 1992 High Press. Res. 10659

Deacon A M, Clark S M and Pattison P 1990 SERC Daresburg Laboratory Report DL/SCI/TM70E

Fuchs H F, Wu D Q and Chu B 1990 Rev. Sci. Instrum. 61712

Gago T, Li Y, Rousseau J, Limliu K and Chu B 1993 Rev. Sci. Instrum. 64390

Godwal B K, Jayaraman A, Meenakshi S, Rao R S, Sikka S K and Vijayakumar V 1997 Phys. Rev. B56 14871

Hamaya N, Okabe N, Yamakata M, Yagi T and Shimomura O 1996 High Press. Res. 14287

Mao H K, Xu J A and Bell P M 1986 J. Geophys. Res. 914673

Pearson W B 1967 in Handbook of lattice spacings and structure of metals and alloys (Oxford: Pergamon Press) Vol. 2

Rietveld H M 1967 Acta Crystallogr. 22151

Rietveld H M 1969 J. Appl. Cryst. 265

Sinha Amar, Bhawe B D, Panchal C G, Shyam A, Srinivasan M and Joshi V M 1996 Nucl. Instrum. Meth. B111 171

Vaidya S N and Kennedy G C 1971 J. Phys. Chem. Solids 32 951 